\title{
Analysis of Interference in Cognitive Radio Networks with Unknown Primary Behavior
}

\author{
Chunxiao Jiang*†, Yan Chen*, K. J. Ray Liu* and Yong Ren ${ }^{\dagger}$ \\ *Department of Electrical and Computer Engineering, University of Maryland, College Park, MD 20742, USA \\ ${ }^{\dagger}$ Tsinghua National Laboratory for Information Science and Technology (TNList) \\ Department of Electronic Engineering, Tsinghua University, Beijing 100084, P. R. China \\ Email: *\{jcx, yan, kjrliu $\} @$ umd.edu, ${ }^{\dagger}$ reny@thu.edu.cn
}

\begin{abstract}
One critical issue in dynamic spectrum access of cognitive radio networks is the analysis of interference caused by Secondary Users (SUs). Most of the current works focus on mitigating the aggregated interference effects of SUs at Primary Users (PUs) in the physical layer. However, the interference is also dynamically related to the communication behaviors between PUs and SUs. In this paper, we analyze the interference caused by SUs in the MAC layer by taking into account the dynamic behaviors between PUs and SUs. Based on the ON-OFF primary channel state model, we derive the close-form expressions for the probability of interference caused by SUs and quantify the interference effect in two scenarios: slotted secondary network and non-slotted secondary network. We also discuss how to control SUs' access behavior such that the normal communication of PUs can be guaranteed. Finally, simulation results are shown to verify the effectiveness of our analysis.
\end{abstract}

\section{INTRODUCTION}

Cognitive radio is considered as an effective approach to mitigate recent problem of crowded electromagnetic radio spectrums. Compared with static spectrum allocation, dynamic spectrum access (DSA) technology can greatly enhance the utilization ratio of the existing spectrum resources [1]. In DSA, secondary users (SUs) can dynamically access the primary users (PUs') spectrum, while normal communication activities in licensed spectrum are not interfered [2].

One of the most important task in the implementation of DSA technology is to avoid SUs adversely interfering the normal communication activities of PUs in licensed bands [3]. One way is to strictly prevent SUs from interfering PUs in both time domain and frequency domain [4], and the other is to allow interference from SUs while minimizing the interference effect to PUs. To overcome the latter issue, the foundation is to model and analyze the interference caused by SUs so as to reveal the quantitative impacts on PUs. Most of the existing works on interference modeling can be summarized into two categories: spatial and accumulated interference modelings [5]. The main idea of the spatial interference modeling is to reveal how the interference caused by SUs may vary with their different spacial positions relative to primary receivers [6][7][8]. While the accumulated interference model focuses on analyzing the accumulated interference power of SUs at primary receiver through adopting different channel fading models such as [9][10] with only exponential path loss, and [11][12] with additional log-normal shadowing.
However, those traditional interference analysis are only based on aggregating SUs' transmission power with different path fading coefficients, regardless of the communication behaviors of PUs and SUs. In this paper, we will study the interference through analyzing the relationship between SUs' dynamic access and the states of primary channels in the MAC layer. Especially, we will concentrate on the situation when SUs are confronted with unknown primary behavior. If SUs have the perfect knowledge of PUs' communication mechanism, the interference is mainly from imperfect sensing which has been studied a lot [13]. Therefore, perfect sensing is assumed in this paper. We model the primary channel state as an ON-OFF process and derive the probability of interference to PUs and quantify the interference effect. Besides, the impact of the interference to PUs' average data rate is also analyzed. Based on these analysis, we further discuss how to control SUs' access time so as to ensure PUs' normal communication.

The rest of this paper is organized as follows. Firstly, system model is showed in Section II. Then, two different scenarios of secondary network are described in Section III. Next, we explicitly derive the expression of interference probability and quantity in Section IV and V respectively. Section VI presents how to control SUs' behavior. Finally, simulation results are shown in Section VII and conclusion is drawn in Section VIII.

\section{SySTEM MOdEL}

\section{A. Network Entity}

In our system, SUs build a half-duplex multi-hop network and dynamically seek for available licensed channels among $N$ primary channels. Here, "half-duplex" not only means that SUs cannot simultaneously transmit and receive data, but also specially refers that SUs cannot perform spectrum sensing while keeping data communication. Another important characteristic of our system is that PUs' communication is private and unknown to SUs.

\section{B. Primary Channel State Model}

Since SUs have no idea about primary communication behavior and hence cannot be synchronous with PUs, there is no concept of "time slot" in the primary channel from the views of SUs. Instead, each primary channel just alternatively switches between the ON and OFF state, as Fig. 1 shows. 


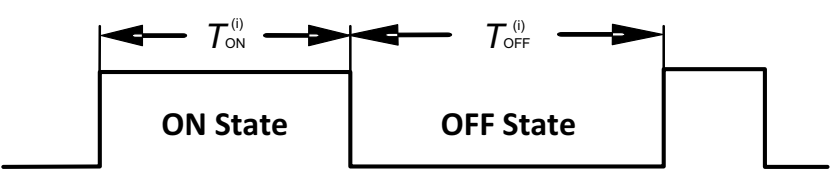

Fig. 1. Illustration of the ON-OFF primary channel state.

The ON state means the channel is being occupied by some PUs, while the OFF state is the "spectrum hole" for SUs where PUs are absent. For channel $i$, the length of the ON(OFF) state denoted by $T_{\mathrm{ON}}^{(\mathrm{i})}\left(T_{\mathrm{OFF}}^{(\mathrm{i})}\right)$ statistically obeys some particular distribution, which depends on the type of primary service, e.g., digital TV broadcasting or cellular communication. For a more general case, we regard that $T_{\text {ON }}^{(\mathrm{i})}\left(T_{\mathrm{OFF}}^{(\mathrm{i})}\right)$ obeys exponential distribution with parameter $\lambda_{1}^{(\mathrm{i})}\left(\lambda_{0}^{(\mathrm{i})}\right)$ as follows

$$
\left\{\begin{array}{l}
T_{\mathrm{ON}}^{(\mathrm{i})} \sim f_{\mathrm{ON}}^{(\mathrm{i})}(t)=\frac{1}{\lambda_{1}^{(i)}} e^{-t / \lambda_{1}^{(\mathrm{i})}} \\
T_{\mathrm{OFF}}^{(\mathrm{i})} \sim f_{\mathrm{OFF}}^{(\mathrm{i})}(t)=\frac{1}{\lambda_{0}^{(\mathrm{ij}}} e^{-t / \lambda_{0}^{(\mathrm{i})}} .
\end{array}\right.
$$

Meanwhile, $\mu_{\mathrm{ON}}^{(\mathrm{i})}=\lambda_{1}^{(\mathrm{i})} /\left(\lambda_{0}^{(\mathrm{i})}+\lambda_{1}^{(\mathrm{i})}\right)$ is defined as the occurrence probability of the ON state in channel $i$, also called as channel utilization ratio. Similarly, $\mu_{\mathrm{OFF}}^{(\mathrm{i})}=1-\mu_{\mathrm{ON}}^{(\mathrm{i})}=\lambda_{0}^{(\mathrm{i})} /\left(\lambda_{0}^{(\mathrm{i})}+\lambda_{1}^{(\mathrm{i})}\right)$ is defined as the occurrence probability of the OFF state in channel $i$. The channel parameters $\lambda_{1}^{(\mathrm{i})}$ and $\lambda_{0}^{(\mathrm{i})}$ can be effectively estimated by a maximum likelihood estimator [14].

\section{SECONDARY USERS' COMMUNiCATION BeHAVIORS}

In this section, we will define the communication behaviors of SUs so as to analyze their interference to PUs. Considering different communication mechanisms of secondary network in the MAC layer, we will study two typical scenarios in this paper: slotted behavior and non-slotted behavior.

\section{A. Slotted Behavior}

In the first scenario, we assume that the system clock of the secondary network is divided into time slots with same length, as shown in Fig. 2-(a) with the example of two primary channels. At the beginning of each slot, SUs sense all primary channels within time $T_{s}$. After sensing, SUs access all available primary channels according to the sensing results. If no available channel is discovered, SUs will keep silent in this slot and wait for the next slot.

In Fig. 2-(a), $T_{a}$ is defined as the length of the access time. Once SUs begin transmitting or receiving data packets, they will no longer be able to perform spectrum sensing during the whole access time $T_{a}$. It is assumed that SUs are always intent to access primary channel, which means as long as there are idle channels, they will access these idle channels. This assumption means that we are analyzing the worst case of the first scenario, or the maximum interference is considered since SUs are always trying to access primary channels.

\section{B. Non-slotted Behavior}

In the second scenario, SUs' communication with each other are not restricted to time slots. Once there are packets need to be transmitted, SUs will begin to search for available primary channels within time $T_{s}$. If no available spectrum is discovered

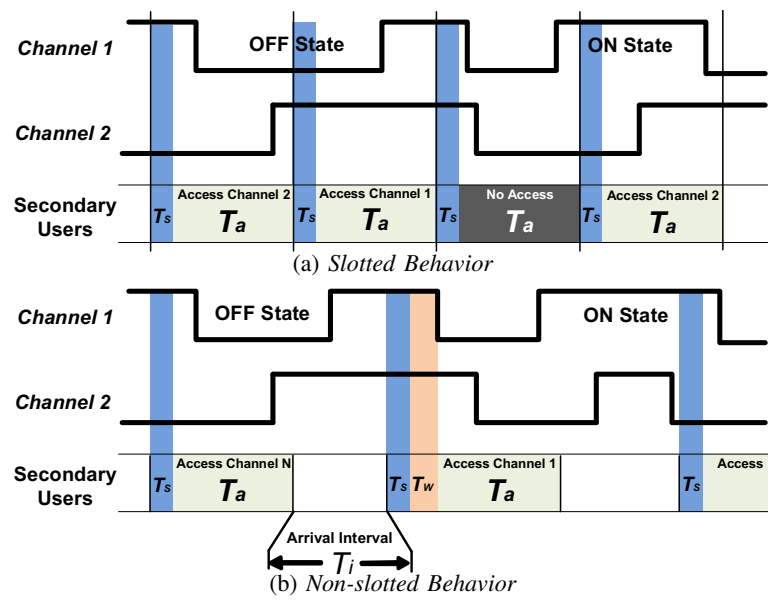

Fig. 2. Illustration of SUs' two communication behaviors.

temporarily, they will keep performing spectrum sensing until some idle channel is found. The length of this waiting time is denoted by $T_{w}$ as shown in Fig. 2-(b). It is assumed that SUs' packets arrive by Poisson process with arrival rate $\lambda_{s}^{-1}$. Thus, the arrival interval $T_{i}$ obeys exponential distribution with parameter $\lambda_{s}$, i.e., $T_{i} \sim f_{T_{i}}(t)=\frac{1}{\lambda_{s}} e^{-t / \lambda_{s}}$ and $\mathbb{E}\left(T_{i}\right)=\lambda_{s}$.

In our model, we assume that SUs' access time $T_{a}$ is less than $\lambda_{1}^{(\mathrm{i})}$ or $\lambda_{0}^{(\mathrm{i})}$, i.e., $T_{a}<\lambda_{1}^{(\mathrm{i})}, \lambda_{0}^{(\mathrm{i})}$, since if the access time can cover several ON and OFF states, the interference caused by SUs will be too severe for PUs. For the sensing time $T_{s}$, since it is too small compared to $T_{a}, T_{w}, \lambda_{1}^{(\mathrm{i})}$ and $\lambda_{0}^{(\mathrm{i})}$, i.e., $T_{s} \ll T_{a}, T_{w}, \lambda_{1}^{(\mathrm{i})}, \lambda_{0}^{(\mathrm{i})}$, we omit $T_{s}$ in the following interference calculations. For these two scenarios, we can see that SUs' access behaviors are two renewal processes. The holding times of these two processes are defined as SUs' access period $T_{p}$, which can be computed as follows

$$
T_{p}=\left\{\begin{array}{l}
T_{s}+T_{a} \approx T_{a} \\
T_{a} \text { or } T_{w}+T_{a} \quad \text { Slotted Behavior, }
\end{array}\right.
$$

In the following sections, we will discuss the interference probability and quantity for each scenario respectively.

\section{Probability of Interference Caused BY SECONDARY USERS}

If SUs can be synchronous with PUs, they can vacate the occupied available channels by the end of the slot. In such a case, the potential interference from SUs only comes from their imperfect spectrum sensing. However, when SUs are confronted with unknown primary channels, additional interferences will appear since SUs may fail to discover PUs' recurrence during their access, as shown by the yellow regions in Fig. 3 with the example of two primary channels. The essential reason is that SUs cannot keep sensing the accessed channel during data transmission or receiving.

The interference caused by SUs happens only when the following three events happen simultaneously

- $e_{1}$ : SUs are intent to access the primary channels;

- $e_{2}$ : SUs access the OFF state of channel $i$;

- $e_{3}$ : PUs come back to channel $i$ before SUs' $T_{a}$ ends. 


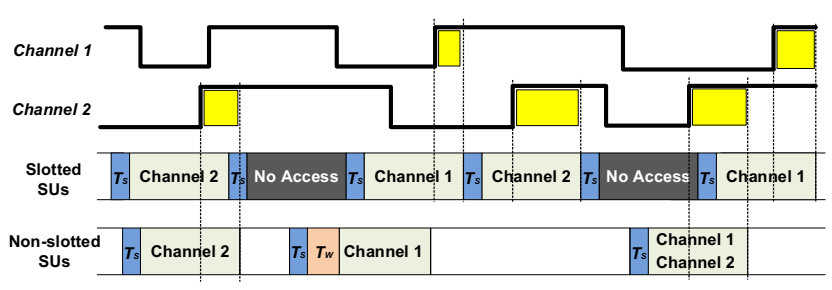

Fig. 3. Examples that SUs fail to discover PUs' recurrence.

Since these three events are mutually independent, the interference probability of channel $i, P_{I}^{(\mathrm{i})}$, can be expressed by

$$
P_{I}^{(\mathrm{i})}=P\left(e_{\mathbf{1}}\right) \cdot P\left(e_{\mathbf{2}}\right) \cdot P\left(e_{\mathbf{3}}\right) .
$$

In the following subsections, we will compute the probability of these three events respectively.

\section{A. Probability of $e_{\mathbf{1}}: P\left(\boldsymbol{e}_{\mathbf{1}}\right)$}

In the scenario of the slotted behavior, SUs are always intent to access primary channels. Therefore, we have

$$
P\left(\boldsymbol{e}_{\mathbf{1}}\right)=1 \quad \text { Slotted Behavior. }
$$

On the other hand, when SUs' behavior is non-slotted, the arrival of SUs' access periods is a Poisson process with time interval $T_{i}$. Therefore, $P\left(\boldsymbol{e}_{\mathbf{1}}\right)$ is the occurrence probability of SUs' access period and can be computed by

$$
P\left(\boldsymbol{e}_{\mathbf{1}}\right)=\frac{\mathbb{E}\left(T_{p}\right)}{\mathbb{E}\left(T_{p}\right)+\mathbb{E}\left(T_{i}\right)} .
$$

From Fig. 2-(b), we can see that $T_{p}=T_{a}$ when at least one idle primary channel is discovered, and $T_{p}=T_{w}+T_{a}$ when there is no available channel. Therefore, $\mathbb{E}\left(T_{p}\right)$ can be calculated as follows

$$
\begin{aligned}
\mathbb{E}\left(T_{p}\right) & =\left(1-\prod_{j=1}^{N} \mu_{\mathrm{oN}}^{(\mathrm{j})}\right) \cdot T_{a}+\prod_{j=1}^{N} \mu_{\mathrm{oN}}^{(\mathrm{j})} \cdot\left(T_{a}+\mathbb{E}\left(T_{w}\right)\right) \\
& =T_{a}+\prod_{j=1}^{N} \mu_{\mathrm{oN}}^{(\mathrm{j})} \cdot \mathbb{E}\left(T_{w}\right)
\end{aligned}
$$

where $\prod_{j=1}^{N} \mu_{\mathrm{oN}}^{(\mathrm{j})}$ represents the probability that all the primary channels are in the ON state.

According to the renewal theory [15], the waiting time $T_{w}$ is the forward recurrence time of the ON state $\widehat{T}_{\mathrm{OFF}}^{(\mathrm{i})}$ and its expectation is $\lambda_{1}^{(j)}$ for channel $i$. Since SUs will access to the channel that first returns to the ON state, which is statistically the channel with least $\lambda_{1}$, thus $\mathbb{E}\left(T_{w}\right)=\hat{\lambda}_{1}=\min _{1 \leq i \leq N} \lambda_{1}^{(i)}$. Therefore, according to (5) and (6), $P\left(\boldsymbol{e}_{\mathbf{1}}\right)$ becomes

$$
P\left(\boldsymbol{e}_{\mathbf{1}}\right)=\frac{T_{a}+\prod_{j=1}^{N} \mu_{\mathrm{ON}}^{(\mathrm{j})} \cdot \hat{\lambda}_{1}}{T_{a}+\prod_{j=1}^{N} \mu_{\mathrm{ON}}^{(\mathrm{j})} \cdot \hat{\lambda}_{1}+\lambda_{s}} \quad \begin{gathered}
\text { Non-slotted } \\
\text { Behavior. }
\end{gathered}
$$

\section{B. Probability of $e_{2}: P\left(e_{2}\right)$}

The probability that SUs access the OFF state $P\left(e_{2}\right)$ is equal to the occurrence probability of the OFF state, i.e.,

$$
P\left(e_{2}\right)=\mu_{\mathrm{OFF}}^{(\mathrm{i})} \quad \text { For Both Behaviors. }
$$

\section{Probability of $e_{3}: P\left(e_{3}\right)$}

The event $e_{3}$ happens only when the forward recurrence time of the OFF state $\widehat{T}_{\mathrm{OFF}}^{(\mathrm{i})}$ is smaller than the access time, i.e., $0 \leq \widehat{T}_{\text {OFF }}^{(\mathrm{i})} \leq T_{a}$. Therefore, for both slotted and non-slotted behaviors, $P\left(e_{3}\right)$ can be computed by

$$
P\left(\boldsymbol{e}_{\mathbf{3}}\right)=P\left(0 \leq \widehat{T}_{\mathrm{OFF}}^{(\mathrm{i})} \leq T_{a}\right) .
$$

Let $\widehat{f}_{\mathrm{OFF}}^{(\mathrm{i})}(t)$ be the p.d.f (probability density function) of $\widehat{T}_{\mathrm{OFF}}^{(\mathrm{i})}$. Then, according to the renewal theory [15], $\widehat{f}_{\mathrm{OFF}}^{(\mathrm{i})}(t)$ is

$$
\widehat{f}_{\mathrm{OFF}}^{(\mathrm{i})}(t)=\frac{1-F_{\mathrm{OFF}}^{(\mathrm{i})}(t)}{\lambda_{0}^{(\mathrm{i})}},
$$

where $F_{\mathrm{OFF}}^{(\mathrm{i})}(t)$ is the $c . d . f$ (cumulative distribution function) of $T_{\text {OFF. }}^{(\mathrm{i})}$. According to (9-10), we can re-write $P\left(\boldsymbol{e}_{\mathbf{3}}\right)$ as

$$
\begin{aligned}
P\left(\boldsymbol{e}_{\mathbf{3}}\right) & =P\left(0 \leq \widehat{T}_{\mathrm{OFF}}^{(\mathrm{i})} \leq T_{a}\right) \\
& =\int_{0}^{T_{a}} \frac{1-F_{\mathrm{OFF}}^{(\mathrm{i})}(t)}{\lambda_{0}^{(\mathrm{i})}} d t \\
& =1-e^{-T_{a} / \lambda_{0}^{(\mathrm{i})}} \quad \text { For Both Behaviors. }
\end{aligned}
$$

By substituting (4), (7), (8) and (11) into (3), we can obtain the close-form expression of $P_{I}^{(\mathrm{i})}$ as follows

$$
P_{I}^{(\mathrm{i})}=\left\{\begin{array}{lc}
\mu_{\mathrm{OFF}}^{(\mathrm{i})}\left(1-e^{-\frac{T_{a}}{\lambda_{0}^{(i)}}}\right) & \begin{array}{ll}
\text { Slotted } \\
\text { Behavior },
\end{array} \\
\frac{\mu_{\mathrm{OFF}}^{(\mathrm{i})}\left(T_{a}+\prod_{j=1}^{N} \mu_{\mathrm{ON}}(\mathrm{j}) \cdot \hat{\lambda}_{1}\right.}{T_{a}+\prod_{j=1}^{N} \mu_{\mathrm{ON}}^{(\mathrm{j})} \cdot \hat{\lambda}_{1}+\lambda_{s}}\left(1-e^{-\frac{T_{a}}{\lambda_{0}^{(i)}}}\right) & \begin{array}{l}
\text { Non-slotted } \\
\text { Behavior. }
\end{array}
\end{array}\right.
$$

\section{Quantity of Interference Caused BY SECONDARY USERS}

In most of the existing works, the quantity of interference was usually measured as the quantity of SUs' signal power at primary receiver in the physical layer. In this paper, we measure the interference quantity based on the communication behaviors of PUs and SUs in the MAC layer. As shown in Fig. 3, the yellow region indicates the interference period in the ON state of channel $i$. We define the quantity of interference as the ration between this interference period and the average length of the ON state, which can be written as follows

$$
Q_{I}^{(\mathrm{i})}=P\left(\boldsymbol{e}_{\mathbf{1}}\right) \cdot \frac{\left.\mathbb{E}\left(T_{a}-\widehat{T}_{\mathrm{OFF}}^{(\mathrm{i})}\right)\right|_{\left[0 \leq \widehat{T}_{\mathrm{OFF}}^{(\mathrm{i})} \leq T_{a}\right]}}{\mathbb{E}\left(T_{\mathrm{ON}}^{(\mathrm{i})}\right)},
$$

where $\left.\mathbb{E}\left(T_{a}-\widehat{T}_{\mathrm{OFF}}^{(\mathrm{i})}\right)\right|_{\left[0 \leq \widehat{T}_{\mathrm{OFF}}^{(\mathrm{i})} \leq T_{a}\right]}$ is the expectation of $T_{a}-$ $\widehat{T}_{\mathrm{OFF}}^{(\mathrm{i})}$ within the interval $0 \leq \widehat{T}_{\mathrm{OFF}}^{(\mathrm{i})} \leq T_{a}$. In this paper, we assume that $T_{a}$ is much smaller than $\lambda_{1}^{(\mathrm{i})}$ and $\lambda_{0}^{(\mathrm{i})}$. Therefore, the probability that there are more than one ON or OFF state in $T_{a}$ is very small and is neglected here. 
Let us define $U_{1}=T_{a}-\widehat{T}_{\mathrm{OFF}}^{(\mathrm{i})}$. According to the $p . d . f$ of $\widehat{T}_{\mathrm{OFF}}^{(\mathrm{i})}$ in (10), we can have the p.d.f of $U_{1}$ as follows

$$
U_{1} \sim \frac{1}{\lambda_{0}^{(\mathrm{i})}} e^{-\frac{T_{a}-u_{1}}{\lambda_{0}^{(i)}}} \quad\left[-\infty \leq u_{1} \leq T_{a}\right] .
$$

Then, let us define $U_{2}=\left.U_{1}\right|_{\left[0 \leq \widehat{T}_{\mathrm{OFF}}^{\mathrm{i}} \leq T_{a}\right]}=\left.U_{1}\right|_{\left[0 \leq u_{1} \leq T_{a}\right]}$. In such a case, the p.d.f of $U_{2}$ can be derived by normalizing the p.d.f of $U_{1}$ in the interval $0 \leq u_{1} \leq T_{a}$ as follows

$$
U_{2} \sim \frac{\frac{1}{\lambda_{0}^{(i)}} e^{-\frac{T_{a}-u_{2}}{\lambda_{0}^{(i)}}}}{\int_{0}^{T_{a}} \frac{1}{\lambda_{0}^{(i)}} e^{-\frac{T_{a}-u_{1}}{\lambda_{0}^{(i)}}} d u_{1}}=\frac{e^{-\frac{T_{a}-u_{2}}{\lambda_{0}^{(i)}}}}{\lambda_{0}^{(\mathrm{i})}\left(1-e^{-\frac{T_{a}}{\lambda_{0}^{(i)}}}\right)} .
$$

Therefore, the expectation of $U_{2}$ can be computed as

$$
\mathbb{E}\left(U_{2}\right)=\frac{T_{a}}{1-e^{-\frac{T_{a}}{\lambda_{0}^{(i)}}}}-\lambda_{0}^{(\mathrm{i})} \cdot
$$

According to (4), (7), (13) and (16), the quantity of interference caused by SUs in channel $i, Q_{I}^{(\mathrm{i})}$, can be re-written as

$$
Q_{I}^{(\mathrm{i})}=\left\{\begin{array}{lc}
\frac{1}{\lambda_{1}^{(\mathrm{i})}}\left(\frac{T_{a}}{1-e^{-\frac{T_{a}}{\lambda_{0}^{(\mathrm{i})}}}}-\lambda_{0}^{(\mathrm{i})}\right) & \begin{array}{c}
\text { Slotted } \\
\text { Behavior },
\end{array} \\
\frac{\left(T_{a}+\prod_{j=1}^{N} \mu_{\mathrm{ON}}^{(\mathrm{j})} \cdot \hat{\lambda}_{1}\right) / \lambda_{1}^{(\mathrm{i})}}{T_{a}+\prod_{j=1}^{N} \mu_{\mathrm{ON}}^{(\mathrm{j})} \cdot \hat{\lambda}_{1}+\lambda_{s}}\left(\frac{T_{a}}{1-e^{\frac{T_{a}}{\lambda_{0}^{(i)}}}}-\lambda_{0}^{(\mathrm{i})}\right) & \begin{array}{l}
\text { Non-slotted } \\
\text { Behavior. }
\end{array}
\end{array}\right.
$$

Based on $Q_{I}^{(\mathrm{i})}$, which represents the ratio of the interference periods to PUs' overall communication time, we can calculate the impact of the interference to PUs' average data rate $R_{\mathrm{av}}$. If there is no interference from SUs, PUs' average data rate is $R_{\mathrm{av}}=\log (1+\mathbb{S N R})$, where $\mathbb{S N R}$ denotes the Signal-toNoise Ratio of primary signals at PUs' receiver. On the other hand, if interference occurs, PUs' average data rate is $R_{\mathrm{av}}=$ $\log \left(1+\frac{\mathbb{S N R}}{\mathbb{N} \mathbb{R}+1}\right)$, where $\mathbb{I N R}$ is the Interference-to-Noise Ratio of secondary signals received by PUs. Therefore, PUs' average data rate of channel $i, R_{\mathrm{av}}^{(\mathrm{i})}$ is

$$
R_{\mathrm{av}}^{(\mathrm{i})}=\left(1-Q_{I}^{(\mathrm{i})}\right) \cdot \log \left(1+\mathbb{S N R}^{(\mathrm{i})}\right)+Q_{I}^{(\mathrm{i})} \cdot \log \left(1+\frac{\mathbb{S N R}^{(\mathrm{i})}}{\mathbb{N N R}^{(\mathrm{i})}+1}\right) .
$$

\section{Controlling of SUs' Access Time}

In Section IV and V, we have analyzed the probability and quantity of interference caused by SUs' slotted and nonslotted access, as well as PUs' average data rate under the interference. Based on these results, in this section, we will discuss how to control SUs' access behavior to ensure PUs' normal communication.

In our system, SUs' access behavior refers to their access time $T_{a}$ after discovering some available channels. To guarantee PUs' regular communication, $T_{a}$ should be appropriately chosen. Obviously, a longer access time $T_{a}$ can help SUs achieve higher data rate. However, a longer $T_{a}$ will also bring more interference to PUs and degrade PUs' average data rate. Therefore, a proper $T_{a}$ should be chosen to balance the trade off between the average data rate of SUs and that of PUs.
To ensure PUs' reliable communication, we introduce two QoS constraints: maximum tolerable interference probability $P_{I}^{\uparrow}$ and minimum average achievable data rate $R_{\mathrm{av}}^{\downarrow}$, to restrict SUs' access time. In such a case, the optimization problem of finding the optimal $T_{a}$ for SUs can be formulated as

$$
\begin{aligned}
& \max . T_{a} \\
& \text { s.t. } \begin{cases}\max _{1 \leq i \leq N} P_{I}^{(\mathrm{i})} \leq P_{I}^{\uparrow} & \min _{1 \leq i \leq N} R_{\mathrm{av}}^{(\mathrm{i})} \geq R_{\mathrm{av}}^{\downarrow} \\
T_{a}<\min _{1 \leq i \leq N} \lambda_{0}^{(\mathrm{i})} & T_{a}<\min _{1 \leq i \leq N} \lambda_{1}^{(\mathrm{i})} .\end{cases}
\end{aligned}
$$

According to (12), (13) and (18), we can see that $P_{I}^{(\mathrm{i})}$ and $Q_{I}^{(\mathrm{i})}$ are increasing functions in terms of $T_{a}$ and $R_{\mathrm{av}}^{(\mathrm{i})}$ is a decreasing function in terms of $T_{a}$. Therefore, the optimization problem in (19) can be solved using Newton's Method [16].

\section{Simulation Results}

\begin{tabular}{|c|c|c|c|c|}
\hline Parameter & Value & & & Description \\
\hline$N$ & 3 & \multicolumn{3}{|r|}{ Number of primary channels } \\
\hline$T_{s}$ & $30 \mathrm{~ms}$ & \multicolumn{3}{|c|}{ Sensing time of each access period } \\
\hline$\lambda_{s}$ & $1 \mathrm{sec}$ & \multicolumn{3}{|c|}{ Average arrival interval of SUs' access periods } \\
\hline $\mathbb{S N R}$ & $5 \mathrm{~dB}$ & \multicolumn{3}{|c|}{ SNR of primary signals at PUs' receiver } \\
\hline $\mathbb{I N \mathbb { R }}$ & $3 \mathrm{~dB}$ & \multicolumn{3}{|c|}{ INR of secondary signals at PUs' receiver } \\
\hline Channel $i$ & 1 & 2 & 3 & Description \\
\hline$\lambda_{1}^{(\mathrm{i})}(\mathrm{sec})$ & 1.5 & 1.8 & 3.6 & Average length of the ON state \\
\hline$\lambda_{0}^{(1)}(\mathrm{sec})$ & 3.5 & 2.2 & 2.6 & Average length of the OFF state \\
\hline
\end{tabular}

In this section, we conduct simulations to verify the effectiveness of our analysis. The parameters used in the evaluation are listed in Table I. We assume that there are 3 primary channels in our simulation.

TABLE I

PARAMETERS FOR PERFORMANCE EVALUATION.

\section{A. Interference Probability $P_{I}^{(i)}$}

With the parameters listed in Table I, the theoretical value of $P_{I}^{(\mathrm{i})}$ can be computed according to (12) and are shown in Fig. 4-(a) and Fig. 5-(a). By simulating PUs' and SUs' behaviors using Matlab and counting the corresponding interference probability, we have the simulation results for $P_{I}^{(\mathrm{i})}$, which are denoted by black lines in the figures. It can be seen that the simulation results of each channel finally match well with the theoretical values after some fluctuations at the beginning. We can also see that the interference of non-slotted behavior is less than that of slotted behavior since a small arrival rate is used in the non-slotted behavior.

\section{B. Interference Quantity $Q_{I}^{(i)}$}

Similar to $P_{I}^{(\mathrm{i})}$, the theoretical value of interference quantity $Q_{I}^{(\mathrm{i})}$ can be found through (17) and are shown in Fig. 4-(b) and Fig. 5-(b). As to the simulation, once the interference occurs, we calculate and record the ratio of the accumulated interference periods to the accumulated periods of the $\mathrm{ON}$ states in each channel. The ratios at different time are illustrated by three black lines in the figures. Similar to that of $P_{I}^{(i)}$, all the simulation results converge to the corresponding theoretical results. Therefore, the close-form expressions in (12) and (17) are very accurate and can be applied to calculate the interference in the practical cognitive radio system. 


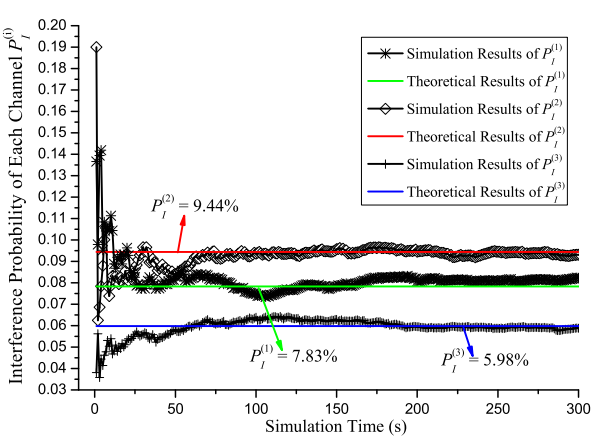

(a) Interference Probability

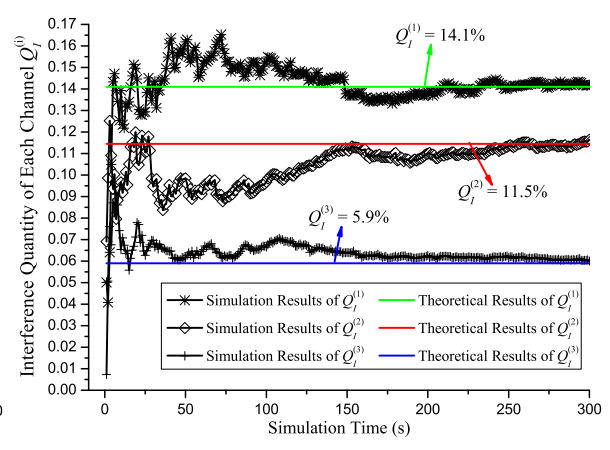

(b) Interference Quantity

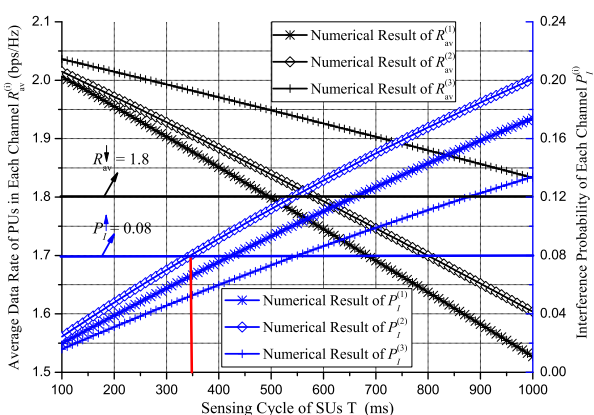

(c) PU's and SUs' Average Data Rate

Fig. 4. Performance of SUs with slotted behavior.

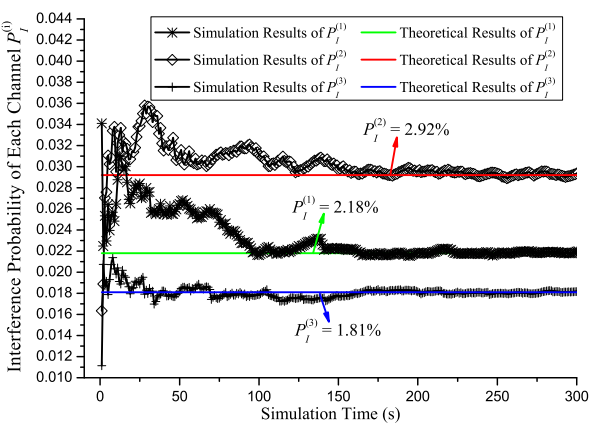

(a) Interference Probability

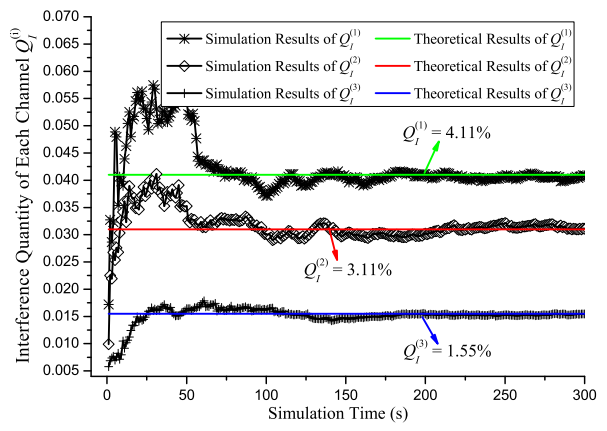

(b) Interference Quantity

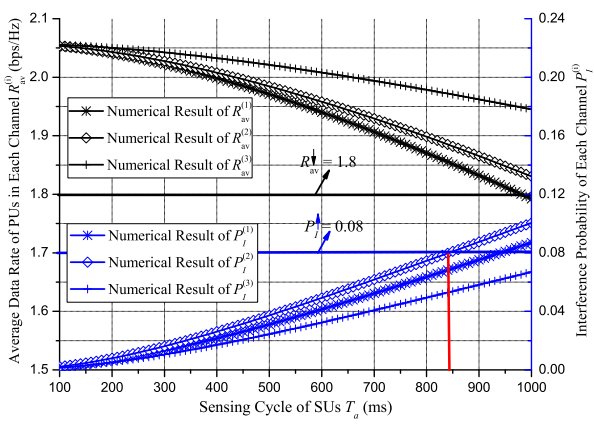

(c) PU's and SUs' Average Data Rate

Fig. 5. Performance of SUs with non-slotted behavior.

\section{SUs' Access Time $T_{a}$}

The simulation results of PUs' average data rate $R_{\mathrm{av}}^{(\mathrm{i})}$ and interference probability $P_{I}^{(\mathrm{i})}$ varying with SUs' sensing cycle $T_{a}$ are shown in Fig. 4-(c) and Fig. 5-(c) separately. We can see that $R_{\mathrm{av}}^{(\mathrm{i})}$ is a decreasing function in terms of $T_{a}$ and $P_{I}^{(\mathrm{i})}$ is an increasing function in terms of $T_{a}$. In order to guarantee PUs' normal communications, we here use $R_{\mathrm{av}}^{\downarrow}=1.8 \mathrm{bps} / \mathrm{Hz}$ and $P_{I}^{\uparrow}=0.08$ as shown in Fig. 4-(c) and Fig. 5-(c) with black and blue horizontal lines. According to the constraints in (19), $T_{a}$ should be no larger than the location of the red vertical lines in Fig. 4-(c) and Fig. 5-(c). Thus, the optimal $T_{a}$ should be around $350 \mathrm{~ms}$ for the slotted behavior and $850 \mathrm{~ms}$ for the non-slotted behavior. When $R_{\mathrm{av}}^{\downarrow}$ and $P_{I}^{\uparrow}$ are given, the value of $T_{a}$ is determined by with the channel parameters $\lambda_{0}^{(\mathrm{i})}$ and $\lambda_{1}^{(\mathrm{i})}$. Therefore, SUs should dynamically adjust $T_{a}$ according to the estimated channel parameters.

\section{CONCLUSION}

In this paper, we discussed the interference caused by slotted and non-slotted SUs based on primary ON-OFF channel model. The impact of the interference to PUs' average data rate is also analyzed. We further discussed how to adjust SUs' access time in order to control the level of interference. In the future work, we will design a cognitive MAC protocol based on the interference analysis of this paper.

\section{REFERENCES}

[1] S. Haykin, "Cognitive radio: brain-empowered wireless communications," IEEE J. Sel. Areas Commun., vol. 23, no. 2, pp. 201-220, 2005.
[2] K. J. R. Liu and B. Wang, Cognitive Radio Networking and Security: A Game Theoretical View. Cambridge University Press, 2010.

[3] B. Wang and K. J. R. Liu, "Advances in cognitive radios: A survey," IEEE J. Sel. Topics Signal Process., vol. 5, no. 1, pp. 5-23, 2011.

[4] B. Wang, Z. Ji, K. J. R. Liu, and T. C. Clancy, "Primary-prioritized markov approach for efficient and fair dynamic spectrum allocation," IEEE Trans. Wireless Commun., vol. 8, no. 4, pp. 1854-1865, 2009.

[5] Z. Chen, C. Wang, X. Hong, J. Thompson, S. A. Vorobyov, and X. Ge, "Interference modeling for cognitive radio networks with power or contention control," in Proc. IEEE WCNC'10.

[6] G. L. Stuber, S. M. Almalfouh, and D. Sale, "Interference analysis of TV band whitespace," Proc. IEEE, vol. 97, no. 4, pp. 741-754, 2009.

[7] M. Vu, D. Natasha, and T. Vahid, "On the primary exclusive regions in cognitive networks," IEEE Trans. Wireless Commun., vol. 8, no. 7, pp. 3380-3385, 2008.

[8] R. S. Dhillon and T. X. Brown, "Models for analyzing cognitive radio interference to wireless microphones in TV bands," in Proc. IEEE DySPAN'08, pp. 1-10.

[9] N. Hoven and A. Sahai, "Power scaling for cognitive radio," in Proc. IEEE WNCMC'05, pp. 250-255.

[10] M. Timmers, S. Pollin, A. Dejonghe, A. Bahai, L. V. Perre, and F. Catthoor, "Accumulative interference modeling for cognitive radios with distributed channel access," in Proc. IEEE CrownCom'08, pp. 1-7.

[11] R. Menon, R. M. Buehrer, and J. H. Reed, "On the impact of dynamic spectrum sharing techniques on legacy radio systems," IEEE Trans. Wireless Commun., vol. 7, no. 11, pp. 4198-4207, 2008.

[12] A. Ghasemi and E. S. Sousa, "Interference aggregation in spectrumsensing cognitive wireless networks," IEEE Journal of Selected Topics in Signal Processing, vol. 2, no. 1, pp. 41-56, 2008.

[13] A. A. El-Sherif and K. J. R. Liu, "Joint design of spectrum sensing and channel access in cognitive radio networks," IEEE Trans. Wireless Commun., vol. 10, no. 6, pp. 1743-1753, 2011.

[14] H. Kim and K. G. Shin, "Efficient discovery of spectrum opportunities with MAC-layer sensing in cognitive radio networks," IEEE Trans. Mobile Computing, vol. 7, no. 5, pp. 533-545, 2008.

[15] D. R. Cox, Renewal Theory. Butler and Tanner, 1967.

[16] D. P. Bertsekas, Nonlinear Programming. Athena Scientific, 1999. 